\title{
Cut-off period for slow magnetoacoustic waves in coronal plasma structures
}

\author{
A. N. Afanasyev ${ }^{1,2, \star}$ and V. M. Nakariakov ${ }^{1,3,4}$ \\ ${ }^{1}$ Centre for Fusion, Space and Astrophysics, Department of Physics, University of Warwick, CV4 7AL, UK \\ e-mail: afa@iszf.irk.ru \\ 2 Institute of Solar-Terrestrial Physics SB RAS, PO Box 291, Lermontov St. 126A, 664033 Irkutsk, Russia \\ 3 School of Space Research, Kyung Hee University, 446-701 Yongin, Gyeonggi, Korea \\ ${ }^{4}$ Central Astronomical Observatory at Pulkovo of the Russian Academy of Sciences, 196140 St. Petersburg, Russia
}

Received 14 May 2015 / Accepted 28 July 2015

ABSTRACT

\begin{abstract}
Context. There is abundant observational evidence of longitudinal compressive waves in plasma structures of the solar corona, which are confidently interpreted in terms of slow magnetoacoustic waves. The uses of coronal slow waves in plasma diagnostics, as well as analysis of their possible contribution to coronal heating and the solar wind acceleration, require detailed theoretical modelling. Aims. We investigate the effects of obliqueness, magnetic field, and non-uniformity of the medium on the evolution of long-wavelength slow magnetoacoustic waves guided by field-aligned plasma non-uniformities, also called tube waves. Special attention is paid to the cut-off effect due to the gravity stratification of the coronal plasma.

Methods. We study the behaviour of linear tube waves in a vertical untwisted straight field-aligned isothermal plasma cylinder. We apply the thin flux tube approximation, taking into account effects of stratification caused by gravity. The dispersion due to the finite radius of the flux tube is neglected. We analyse the behaviour of the cut-off period for an exponentially divergent magnetic flux tube filled in with a stratified plasma. The results obtained are compared with the known cases of the constant Alfven speed and the pure acoustic wave.

Results. We derive the wave equation for tube waves and reduce it to the form of the Klein-Gordon equation with varying coefficients, which explicitly contains the cut-off frequency. The cut-off period is found to vary with height, decreasing significantly in the low-beta plasma and in the plasma with the beta of the order of unity. The depressions in the cut-off period profiles can affect the propagation of longitudinal waves along coronal plasma structures towards the higher corona and can form coronal resonators.
\end{abstract}

Key words. magnetohydrodynamics (MHD) - waves - Sun: corona - methods: analytical

\section{Introduction}

The abundant observational evidence for magnetohydrodynamic (MHD) wave processes operating in the solar corona motivates the development of precise theoretical models. The importance of studies of coronal waves and oscillations is traditionally associated with such fundamental solar physics problems as coronal heating and solar wind acceleration. The understanding of MHD waves can also give insight into the nature of coronal mass ejections and flares as the wave behaviour may reflect their triggering mechanisms (e.g. Vršnak \& Cliver 2008). Of special importance are seismological applications of wave studies, which provide the valuable and sometimes unique information about important parameters of the solar coronal plasma (Stepanov et al. 2012; De Moortel \& Nakariakov 2012).

Observations in extreme ultraviolet (EUV) spectral lines show the presence in the corona of MHD waves of different types and periods. In particular, the longitudinal along the field, propagating EUV disturbances observed in various solar plasma structures, for example in coronal loops and plumes (e.g. DeForest \& Gurman 1998; Berghmans \& Clette 1999; De Moortel et al. 2000, see also De Moortel 2009, for review) are interpreted in terms of slow magnetoacoustic waves (e.g. Ofman et al. 2000; Nakariakov et al. 2000; Afanasyev \& Nakariakov 2015, see Nakariakov 2006, for review). Their periods are found to range from several minutes to several tens of minutes. For example,

\footnotetext{
^ Visiting Fellow at CFSA, University of Warwick, UK.
}

wave trains of 3 and 5 min periods were observed in coronal loops (De Moortel et al. 2002). The longer longitudinal waves (10-12 min) were detected in polar plumes (Ofman et al. 1997). Yuan et al. (2011) revealed the long-period waves of about 29, 53, and $75 \mathrm{~min}$ in the lower corona. Recently, Bakunina et al. (2013) and Chorley et al. (2010) reported on the long-period oscillations in radio band in sunspot magnetospheres.

With respect to long-period slow magnetoacoustic waves, the solar atmosphere is an essentially dispersive medium. In addition to the dispersion connected with the field-aligned filamentation (Zajtsev \& Stepanov 1975; Edwin \& Roberts 1983), the dispersion effects are also due to gravitational stratification, and result in the cut-off effect for harmonic waves as well as spreading and appearance of the trailing oscillatory wake for broadband pulses. The dispersive behaviour of acoustic waves propagating vertically upwards in the stratified atmosphere was demonstrated by Lamb (1932). Wave equations governing the wave propagation and evolution can be reduced to the Klein-Gordon (KG) equation, which explicitly contains the cut-off frequency.

The behaviour of long-period longitudinal waves in the solar atmosphere in terms of acoustic waves was very intensively investigated several decades ago (see e.g. Sutmann et al. 1998, and references therein). However, one should take into account the magnetic nature of waves as well as the field-aligned structuring of the coronal plasma (e.g. Van Doorsselaere et al. 2008). In such a case, the slow magnetoacoustic mode manifests itself as a tube mode due to the wave-guiding conditions within plasma structures. Because of the field-aligned non-uniformity, the local 
wave vector is essentially oblique with respect to the magnetic field. In low- $\beta$ plasmas, the speed of propagation of tube waves (tube speed) is only slightly different from the sound speed. The effect becomes much more pronounced in plasmas with $\beta \sim 1$. In this context long-period tube waves in solar plasma structures were analysed by Defouw (1976), Roberts \& Webb (1978, 1979), Rae \& Roberts (1982), Hasan \& Kalkofen (1999) and Musielak \& Ulmschneider (2003). These studies were mainly aimed at the case of photospheric flux tubes expanding into the chromosphere. They obtained the KG equation describing the tube wave evolution in the presence of gravitational stratification, and estimated the cut-off frequency. However, the authors considered the case of an exponentially divergent magnetic flux tube with the characteristic scale height equal to twice the density scale height, and therefore they analysed the particular case of a constant Alfvén speed. As a result, they obtained the KG equation with constant coefficients, also assuming a constant sound speed. In a real solar atmosphere, the divergence of magnetic flux tubes may not be linked that way to the density scale height, and the Alfvén speed profile may vary along the magnetic field. Thomas (1982) considered the cut-off frequency in the stratified atmosphere with the horizontal magnetic field. Roberts (2006) raised a new formulation of the problem for longitudinal waves and considered that the stratified atmosphere is penetrated by a uniform vertical magnetic field.

In this paper, we consider the propagation of long-period slow magnetoacoustic waves in coronal plasma structures, taking into account the arbitrary variation (within our simplifying assumptions) of the magnetic field. We utilise the thin flux tube approximation in order to derive the KG equation for tube waves and obtain the cut-off period profiles. The paper is organised as follows. In Sect. 2 the wave equation for tube waves is derived. In Sect. 3 we reduce the wave equation to the $\mathrm{KG}$ form, obtain the cut-off frequency, and analyse its behaviour under coronal conditions. Section 4 contains the discussion of results obtained and concluding remarks.

\section{Wave equation for tube waves}

We consider azimuthally symmetric linear slow magnetoacoustic waves in a vertical magnetic flux tube without steady plasma flows. Assuming that the wavelength is much longer than the radius of the tube, we apply the traditional thin flux tube approximation (e.g. Roberts \& Webb 1978, see also Zhugzhda 1996), using the cylindrical coordinates $(r, \varphi, z)$. The axisymmetric flux tube is assumed to be straight, untwisted, and non-rotating, and filled in with plasma of a constant temperature. The plasma inside the flux tube is stratified by gravity in the vertical direction along the tube axis. We neglect dispersion effects caused by the finite radius of the flux tube (e.g. Zhugzhda 1996) and the finite scale of the wave localisation outside the flux tube (e.g. Roberts 1985; Zhugzhda \& Goossens 2001), therefore analysing sufficiently long waves. The governing set of equations is

$$
\begin{aligned}
& \rho\left(\frac{\partial u}{\partial t}+u \frac{\partial u}{\partial z}\right)=-\frac{\partial p}{\partial z}-\rho g, \\
& \frac{\partial s}{\partial t}+u \frac{\partial s}{\partial z}=0, \\
& p+\frac{B^{2}}{8 \pi}=p_{\mathrm{T}}^{\mathrm{ext}}, \\
& \frac{\partial B}{\partial t}+u \frac{\partial B}{\partial z}+2 B v=0, \\
& \frac{\partial \rho}{\partial t}+2 \rho v+\frac{\partial}{\partial z}(\rho u)=0, \\
& p=p(\rho, s),
\end{aligned}
$$

where $B$ is the longitudinal component of the magnetic field; $v$ is the radial derivative of the radial component of the plasma velocity; $u$ is the longitudinal component of the plasma velocity; $\rho$, $p$, and $s$ are the plasma density, pressure, and specific entropy, respectively; and $g$ is the gravity acceleration. The last equation is the equation of state. Equations (1) take into account the wave-guiding nature of the wave propagation, perturbations of the magnetic field, and gravity stratification of the plasma. The external total pressure $p_{\mathrm{T}}^{\text {ext }}$ is assumed to be constant and therefore we do not consider disturbances in the external medium, concentrating instead on waves inside the flux tube. This assumption is justified if the characteristic wave speed inside the flux tube is lower than the propagation speeds of waves in the external medium (see Roberts \& Webb 1979; Zhugzhda 1996).

Equations (1) are linearised in the standard manner. Small perturbations of the equilibrium state (indicated by the subscript 0) are introduced as follows:

$$
\begin{array}{lll}
\rho=\rho_{0}+\rho_{1}, & p=p_{0}+p_{1}, & s=s_{0}+s_{1}, \\
B=B_{0}+B_{1}, \quad v=v_{1}, & u=u_{1} .
\end{array}
$$

Perturbations of the azimuthal components of the plasma velocity and magnetic field are assumed to be zero since we do not consider the torsional mode in this study. We note that we omit the subscript 1 for the velocity components in the remainder of the paper. The equilibrium values depend on the vertical coordinate $z$.

By substituting Eqs. (2) into (1) and restricting our attention to linear terms only, and also eliminating the variables $s_{1}, p_{1}$, and $v$, we have

$$
\begin{aligned}
& \frac{B_{0}}{4 \pi} \frac{\partial B_{1}}{\partial t}+c_{0}^{2} \frac{\partial \rho_{1}}{\partial t}-\frac{p_{0}}{R_{\mathrm{gas}}}(\gamma-1) u \frac{\mathrm{d} s_{0}}{\mathrm{~d} z}=0 \\
& \rho_{0} \frac{\partial u}{\partial t}+\rho_{1} g-\frac{B_{0}}{4 \pi} \frac{\partial B_{1}}{\partial z}-\frac{B_{1}}{4 \pi} \frac{\mathrm{d} B_{0}}{\mathrm{~d} z}=0 \\
& \rho_{0} \frac{\partial B_{1}}{\partial t}+\rho_{0} \frac{\mathrm{d} B_{0}}{\mathrm{~d} z} u-B_{0} \frac{\partial \rho_{1}}{\partial t}-B_{0} \frac{\mathrm{d} \rho_{0}}{\mathrm{~d} z} u-B_{0} \rho_{0} \frac{\partial u}{\partial z}=0
\end{aligned}
$$

where $c_{0}^{2}=\gamma p_{0} / \rho_{0}$ is the equilibrium sound speed, $R_{\text {gas }}$ is the gas constant, and $\gamma$ is the adiabatic index, which come from the equation of state.

By differentiating Eqs. (3) with respect to $t$ and $z$ and collecting terms with variable $u$ and with its derivatives, we obtain the wave equation

$$
\begin{aligned}
\frac{\partial^{2} u}{\partial t^{2}} & -c_{\mathrm{T}}^{2} \frac{\partial^{2} u}{\partial z^{2}}+\left(\frac{1}{B_{0}} \frac{\mathrm{d} B_{0}}{\mathrm{~d} z} c_{\mathrm{T}}^{2} \frac{V_{\mathrm{A}}^{2}-c_{0}^{2}}{V_{\mathrm{A}}^{2}+c_{0}^{2}}+\gamma g \frac{c_{\mathrm{T}}^{4}}{c_{0}^{4}}\right) \frac{\partial u}{\partial z} \\
& +\left[c_{\mathrm{T}}^{2} \frac{1}{B_{0}} \frac{\mathrm{d}^{2} B_{0}}{\mathrm{~d} z^{2}}+c_{\mathrm{T}}^{2} \frac{1}{B_{0}^{2}}\left(\frac{\mathrm{d} B_{0}}{\mathrm{~d} z}\right)^{2} \frac{c_{0}^{2}-V_{\mathrm{A}}^{2}}{V_{\mathrm{A}}^{2}+c_{0}^{2}}+\frac{1}{B_{0}} \frac{\mathrm{d} B_{0}}{\mathrm{~d} z} \frac{2 g c_{\mathrm{T}}^{2}}{V_{\mathrm{A}}^{2}+c_{0}^{2}}\right. \\
& \left.+\frac{1}{B_{0}} \frac{\mathrm{d} B_{0}}{\mathrm{~d} z} \gamma g \frac{c_{\mathrm{T}}^{2}}{c_{0}^{2}}\left(\frac{1}{\gamma}-\frac{c_{\mathrm{T}}^{2}}{c_{0}^{2}}\right)+N^{2}+\frac{c_{\mathrm{T}}^{2}}{c_{0}^{2}} \frac{g}{H}\left(\frac{1}{\gamma}-\frac{c_{\mathrm{T}}^{2}}{c_{0}^{2}}\right)\right] u=0,
\end{aligned}
$$

where we have introduced the Brunt-Väisälä (buoyancy) frequency $N$, tube speed $c_{\mathrm{T}}$, and density scale height $H$ as

$$
N^{2}=g\left(\frac{1}{\gamma p_{0}} \frac{\mathrm{d} p_{0}}{\mathrm{~d} z}-\frac{1}{\rho_{0}} \frac{\mathrm{d} \rho_{0}}{\mathrm{~d} z}\right), c_{\mathrm{T}}=\frac{c_{0} V_{\mathrm{A}}}{\sqrt{c_{0}^{2}+V_{\mathrm{A}}^{2}}}, \frac{1}{H}=-\frac{1}{\rho_{0}} \frac{\mathrm{d} \rho_{0}}{\mathrm{~d} z},
$$

respectively, and where $V_{\mathrm{A}}=B_{0} / \sqrt{4 \pi \rho_{0}}$ is the equilibrium Alfvén speed. 
Equation (4) describes the dynamics of longitudinal waves in thin magnetic flux tubes. It is useful to consider the limiting cases for plasma conditions inside the flux tube. In the infinite magnetic field limit, $\left(V_{\mathrm{A}} / c_{0} \rightarrow \infty\right)$, the tube speed $c_{\mathrm{T}}$ becomes equal to the sound speed $c_{0}$. In this case, plasma motions in the wave become exactly longitudinal, and the tube wave degenerates into the plane pure acoustic wave. Thus, the effect of the plasma structuring vanishes and Eq. (4) takes the form of the well-known wave equation for acoustic waves in a stratified isothermal medium (see e.g. Sutmann et al. 1998)

$\frac{\partial^{2} u}{\partial t^{2}}-c_{0}^{2} \frac{\partial^{2} u}{\partial z^{2}}+\gamma g \frac{\partial u}{\partial z}=0$,

provided that the vertical gradient of the magnetic field, $\mathrm{d} B_{0} / \mathrm{d} z$, is limited (i.e. not tending to infinity), which is the case, for example for a cylindrical flux tube with a constant magnetic field.

For a thin flux tube with a finite constant magnetic field, $B_{0}=$ const., we have

$\frac{\partial^{2} u}{\partial t^{2}}-c_{\mathrm{T}}^{2} \frac{\partial^{2} u}{\partial z^{2}}+\gamma g \frac{c_{\mathrm{T}}^{4}}{c_{0}^{4}} \frac{\partial u}{\partial z}+\left[N^{2}+\frac{c_{\mathrm{T}}^{2}}{c_{0}^{2}} \frac{g}{H}\left(\frac{1}{\gamma}-\frac{c_{\mathrm{T}}^{2}}{c_{0}^{2}}\right)\right] u=0$.

After some algebra, Eq. (7) completely coincides with Eq. (3.9) of Roberts (2006).

\section{Klein-Gordon form of the wave equation and the cut-off period}

Equation (4) can be reduced to the form of the KG equation. We denote the coefficients in front of $\partial u / \partial z$ and $u$ in Eq. (4) by $K_{1}$ and $K_{2}$, respectively, and introduce the new variable $U$ as

$u=\mathrm{e}^{\Psi(z)} U(z), \quad \Psi^{\prime}=\frac{K_{1}}{2 c_{\mathrm{T}}^{2}}$,

where

$K_{1}=\frac{1}{B_{0}} \frac{\mathrm{d} B_{0}}{\mathrm{~d} z} c_{\mathrm{T}}^{2} \frac{V_{\mathrm{A}}^{2}-c_{0}^{2}}{V_{\mathrm{A}}^{2}+c_{0}^{2}}+\gamma g \frac{c_{\mathrm{T}}^{4}}{c_{0}^{4}}$.

Henceforth, the dash means differentiation with respect to $z$. By substituting Eqs. (8) into (4), we obtain the KG equation for the variable $U$

$\frac{\partial^{2} U}{\partial t^{2}}-c_{\mathrm{T}}^{2} \frac{\partial^{2} U}{\partial z^{2}}+\omega_{\mathrm{u}}^{2} U=0$,

where the squared cut-off frequency, $\omega_{\mathrm{u}}^{2}$, is

$$
\begin{aligned}
\omega_{\mathrm{u}}^{2}= & -c_{\mathrm{T}}^{2}\left(\psi^{\prime}\right)^{2}-c_{\mathrm{T}}^{2} \Psi^{\prime \prime}+K_{1} \Psi^{\prime}+K_{2} \\
= & \frac{c_{\mathrm{T}}^{2}}{4}\left(\frac{1}{B_{0}} \frac{\mathrm{d} B_{0}}{\mathrm{~d} z} \frac{V_{\mathrm{A}}^{2}-c_{0}^{2}}{V_{\mathrm{A}}^{2}+c_{0}^{2}}+\frac{1}{H} \frac{c_{\mathrm{T}}^{2}}{c_{0}^{2}}\right)^{2} \\
& -\frac{c_{\mathrm{T}}^{2}}{2}\left(\frac{1}{B_{0}} \frac{\mathrm{d} B_{0}}{\mathrm{~d} z} \frac{V_{\mathrm{A}}^{2}-c_{0}^{2}}{V_{\mathrm{A}}^{2}+c_{0}^{2}}+\frac{1}{H} \frac{c_{\mathrm{T}}^{2}}{c_{0}^{2}}\right)^{\prime}+K_{2},
\end{aligned}
$$

and

$$
\begin{aligned}
K_{2}= & c_{\mathrm{T}}^{2} \frac{1}{B_{0}} \frac{\mathrm{d}^{2} B_{0}}{\mathrm{~d} z^{2}}+c_{\mathrm{T}}^{2} \frac{1}{B_{0}^{2}}\left(\frac{\mathrm{d} B_{0}}{\mathrm{~d} z}\right)^{2} \frac{c_{0}^{2}-V_{\mathrm{A}}^{2}}{V_{\mathrm{A}}^{2}+c_{0}^{2}}+\frac{1}{B_{0}} \frac{\mathrm{d} B_{0}}{\mathrm{~d} z} \frac{2 g c_{\mathrm{T}}^{2}}{V_{\mathrm{A}}^{2}+c_{0}^{2}} \\
& +\frac{1}{B_{0}} \frac{\mathrm{d} B_{0}}{\mathrm{~d} z} \gamma g \frac{c_{\mathrm{T}}^{2}}{c_{0}^{2}}\left(\frac{1}{\gamma}-\frac{c_{\mathrm{T}}^{2}}{c_{0}^{2}}\right)+N^{2}+\frac{c_{\mathrm{T}}^{2}}{c_{0}^{2}} \frac{g}{H}\left(\frac{1}{\gamma}-\frac{c_{\mathrm{T}}^{2}}{c_{0}^{2}}\right) .
\end{aligned}
$$

Equation (10) gives the explicit expression for the cut-off frequency with an account of the wave-guiding nature of the wave propagation and magnetic field variation. We note that we refer to the quantity expressed by Eq. (10) as "cut-off frequency"; however, it depends on the vertical coordinate $z$ and therefore its role is not as straightforward as for the simpler acoustic case of an isothermal medium when it is a constant. The quantity in Eq. (10) corresponds to the cut-off frequency in a local approximation (see also Sect. 4).

In the limit of the infinite magnetic field, from Eq. (10) we have the usual cut-off frequency for acoustic waves

$\omega_{\mathrm{u}}^{2}=\left(\frac{c_{0}}{2 H}\right)^{2}$

if the gradient of the magnetic field, $\partial B_{0} / \partial z$, is limited. In the case of constant magnetic field, the cut-off frequency coincides with that obtained for the isothermal plasma from Roberts (2006)

$\omega_{\mathrm{u}}^{2}=\frac{c_{\mathrm{T}}^{2}}{4 H^{2}}\left(\frac{c_{\mathrm{T}}}{c_{0}}\right)^{4}-\frac{c_{\mathrm{T}}^{2}}{2 H} \frac{\left(c_{\mathrm{T}}^{2}\right)^{\prime}}{c_{0}^{2}}+N^{2}+\frac{c_{\mathrm{T}}^{2}}{c_{0}^{2}} \frac{g}{H}\left(\frac{1}{\gamma}-\frac{c_{\mathrm{T}}^{2}}{c_{0}^{2}}\right)$.

In the case of an exponentially divergent magnetic flux tube, with the characteristic scale height for the magnetic field equal to twice the density scale height, the Alfvén speed is constant, and we have for the constant cut-off frequency

$\omega_{\mathrm{u}}^{2}=N^{2}+\frac{c_{\mathrm{T}}^{2}}{H^{2}}\left(\frac{3}{4}-\frac{1}{\gamma}\right)^{2}$,

which is consistent with results by Defouw (1976), Roberts \& Webb (1978), Rae \& Roberts (1982) and Musielak \& Ulmschneider (2003). In the limit of the infinite magnetic field, Eq. (13) differs from the cut-off frequency for pure acoustic waves propagating vertically upwards (Eq. (11)). The reason for this appears to be the divergence of the magnetic flux tube as well as its elasticity due to its magnetic nature. The contribution of the geometry and elasticity of the tube to the cut-off effect was discussed in the review by Roberts (1991).

We analyse the behaviour of the cut-off frequency given by Eq. (10). We consider the exponentially divergent magnetic flux tube, $B_{0}=B_{00} \exp (-z / L)$, with different values of the magnetic field scale height, $L$, in order to demonstrate the main differences between our results on the one hand, and the pure acoustic case and the constant Alfvén speed case on the other. The latter cases have been generally used for the interpretation of observations. The number density is assumed to be barometric, $n_{0}=$ $n_{00} \exp (-z / H)$, where $n_{00}=5 \times 10^{8} \mathrm{~cm}^{-3}, H=2 R_{\mathrm{gas}} T / \widetilde{m} M_{\mathrm{H}} g$ is the density scale height, $T=1.4 \times 10^{6} \mathrm{~K}$ is the plasma temperature, $M_{\mathrm{H}}$ is the molar mass of hydrogen, and $\widetilde{m}=1.27$ is the average atomic weight of an ion, and $\gamma=5 / 3$.

For convenience, we plot the cut-off period, which is $2 \pi / \omega_{\mathrm{u}}$. Figure 1 shows the behaviour of the cut-off period with height. Different panels correspond to different values of the magnetic field, $B_{00}$ : (a) $B_{00}=0.5 \mathrm{G}, V_{\mathrm{A}}=43 \mathrm{~km} \mathrm{~s}^{-1}$; (b) $B_{00}=1.5 \mathrm{G}$, $V_{\mathrm{A}}=130 \mathrm{~km} \mathrm{~s}^{-1} ;$ (c) $B_{00}=5.0 \mathrm{G}, V_{\mathrm{A}}=433 \mathrm{~km} \mathrm{~s}^{-1}$; and $(d)$ $B_{00}=10 \mathrm{G}, V_{\mathrm{A}}=866 \mathrm{~km} \mathrm{~s}^{-1}$; the sound speed is constant for all the panels, $c_{0}=175 \mathrm{~km} \mathrm{~s}^{-1}$. Case $(a)$ is likely to correspond to an Alfvén speed that is too low for coronal conditions; however, we should include it to provide the analysis of the high- $\beta$ plasma. Moreover, such plasma conditions could appear, for example in polar plumes at greater heights.

In the case of a weakly divergent magnetic flux tube in comparison with the density fall-off, $L=5 H$ (blue curve in Fig. 1), 

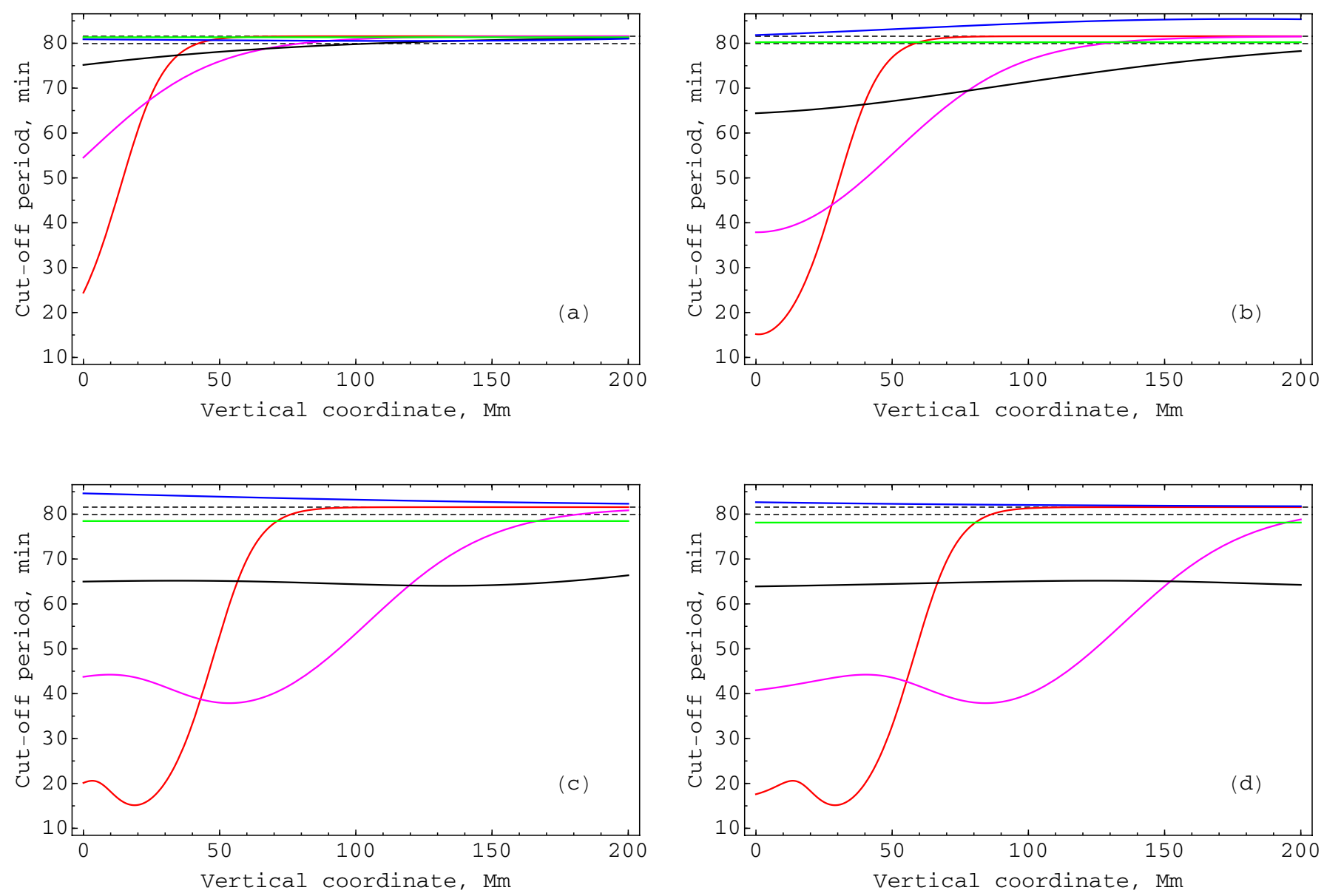

Fig. 1. Dependence of the cut-off period for longitudinal waves in an exponentially divergent magnetic flux tube on the vertical coordinate $z$. The colours correspond to different values of the magnetic field scale height, $L=0.2 H$ (red), $0.5 H$ (purple), $H$ (black), $2 H$ (green), $5 H$ (blue). The dashed lines mark the acoustic cut-off period (lower) and Brunt-Väisälä period (upper). The panels show the cut-off periods for the high a), of the order of unity b), low c), and very low d) plasma $\beta$ in the solar corona (see the corresponding values of the Alfvén speed and sound speed in the text).

the difference in the cut-off period is quite insignificant, reaching as much as about $6 \%$ in the plasma with $\beta \sim 1$. For the coronal conditions $(\beta \lesssim 1)$, the cut-off period for waves in the weakly divergent tube is higher than the acoustic cut-off period and buoyancy period marked by dashed lines in the figure.

The case of $L=2 H$ (green curve in Fig. 1) is the case of the constant Alfvén and sound speeds. The cut-off period is also constant, and its value varies slightly depending on the plasma $\beta$ (Fig. 2).

For $L=H$ (black curve in Fig. 1) in the low- $\beta$ plasma, the cut-off period remains almost constant; however, its value is about $20 \%$ below the acoustic cut-off period. In the plasma with $\beta \sim 1$, the cut-off period grows with height, approaching the acoustic cut-off period above the height of about $200 \mathrm{Mm}$.

Of interest is the behaviour of the cut-off period in strongly divergent magnetic flux tubes with $L=0.2 \mathrm{H}$ and $L=0.5 \mathrm{H}$ (red and purple curves, respectively). The cut-off period is decreased to $20-40 \mathrm{~min}$ at the base of the corona, and then grows to the acoustic cut-off period value. In the low- $\beta$ conditions, the cutoff period profiles have the local maxima lower in the corona, seen in Fig. 1, e.g. for the waves of periods of 15-20 min for the $L=0.2 \mathrm{H}$ case and of about $40 \mathrm{~min}$ for the $L=0.5 \mathrm{H}$ case. In addition, the quite extensive (up to the height of about 50$100 \mathrm{Mm}$ ) plateaus of the reduced cut-off period exist in the low- $\beta$ cases.

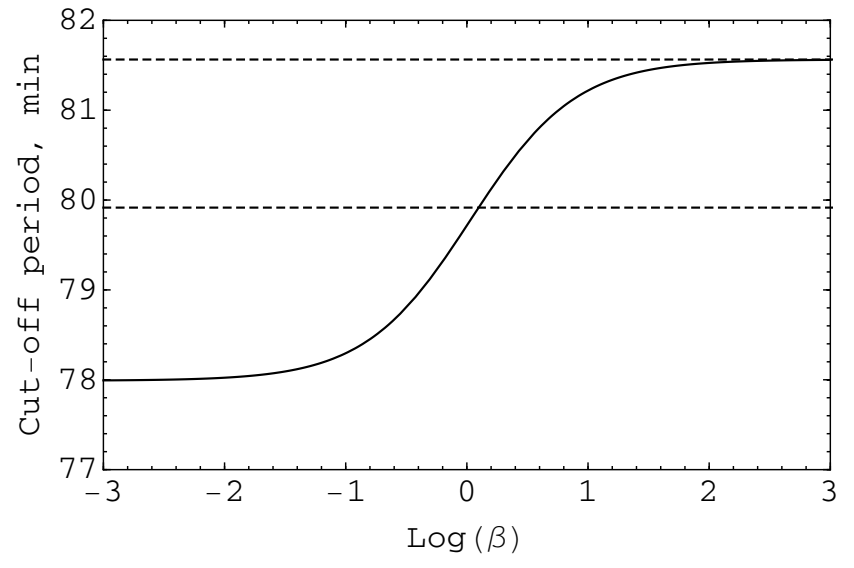

Fig. 2. Dependence of the cut-off period on plasma $\beta$ for the case of a constant Alfvén speed inside the flux tube $(L=2 H)$. The dashed lines mark the acoustic cut-off period (lower) and Brunt-Väisälä period (upper).

\section{Discussion and conclusions}

The behaviour of waves observed in the solar corona can be considerably affected by the transverse non-uniformity of the plasma. In this study, we have constructed the mathematical model describing the dynamics of longitudinal waves in vertical 
field-aligned coronal plasma structures. We have derived the wave equation (Eq. (4)) that could be rewritten as the KleinGordon equation (Eq. (9)), which allows one to consider the propagation and evolution of linear long-wavelength slow magnetoacoustic waves in thin magnetic flux tubes filled in with an isothermal stratified plasma.

The main purpose of the study was to investigate the effect of obliqueness of the slow MHD waves on their evolution in the stratified atmosphere. Waves with wavelengths much greater than the transverse spatial size of the waveguide are essentially oblique. In contrast with plane acoustic waves traditionally used in modelling longitudinal waves in coronal plasma structures, oblique slow magnetoacoustic waves perturb not only the plasma velocity along the field and the density, but also the transverse components of the velocity and the magnetic field. For clarity, long-wavelength slow magnetoacoustic waves guided by fieldaligned plasma non-uniformities are referred to as tube waves.

To analyse the propagation of tube waves in divergent flux tubes, we have used the thin flux tube approximation. Despite the cases of the strong exponential divergence of the tubes, the method appears to be valid for our analysis. Indeed, it is possible to obtain simple estimates in the framework of the exponential model. The transverse size of coronal plasma structures at the base of corona is of the order of $1 \mathrm{Mm}$. At a height of two or four characteristic scale heights for the magnetic field, $L$, the transverse size of a plasma structure grows as much as $e$ or $e^{2}$ times, respectively, and therefore it remains much less (or less, at least) then the wavelength of coronal longitudinal disturbances under study. The latter value can be estimated as $60-120 \mathrm{Mm}$ for the sound speed of $200 \mathrm{~km} \mathrm{~s}^{-1}$ and the periods ranging in 300-600 s.

The stratification of the plasma due to gravity results in the dispersive cut-off effect for harmonic longitudinal waves propagating along the magnetic field upwards. For uniform profiles of the characteristic wave propagation speeds, waves with periods shorter than the cut-off period can propagate freely upwards, while those with longer periods exponentially decay. Thus, the cut-off effect prevents the propagation of longer waves towards the higher corona. The analysis of Eq. (10) has shown that in general the cut-off period varies with height, decreasing significantly in the low- $\beta$ plasma and in the plasma with $\beta \sim 1$. The effect of the varying cut-off period is not as simple as in the case of uniform characteristic wave speeds. Our results are obtained in a local approximation and are valid for sufficiently smooth profiles of the characteristic wave propagation speeds. The depressions in the cut-off period profiles can affect the propagation of longer longitudinal waves along coronal plasma structures in the same way and can prevent their penetration higher in the corona (see e.g. Botha et al. 2011). However, it should be noted that this effect appears to be considerable only for sufficiently extensive plateaus of the reduced cut-off period, while through the quite narrow regions of the reduced cut-off period, waves may tunnel (see e.g. the discussion in Verwichte et al. 2006). On the other hand, in the low- $\beta$ conditions, the local maxima in the cut-off period profiles, found to be situated in the lower corona, imply the possible existence of coronal resonators for longitudinal waves. The detailed analysis of this effect by solving numerically the obtained wave equation, Eq. (4), as well as its implications for the evolution of coronal longitudinal waves will be addressed in a dedicated study.

The effect of the depression of the cut-off period is found to be significant for strongly divergent magnetic flux tubes. A high level of divergence of some coronal plasma structures is indeed observed. In particular, Deforest et al. (1997) found a super-radial expansion of coronal plumes. In addition, observational studies have shown the importance of the geometrical factor on the evolution of waves. For instance, Marsh et al. (2011) pointed out that the flux-tube area divergence had the dominant effect over thermal conduction on oscillations with longer periods (12 min) that travel along cool loops.

The reduced slow magnetoacoustic cut-off period in the corona can be responsible for the appearance of longer-period compressive waves and oscillations observed in the corona. In the case of a uniform profile of the characteristic wave propagation speed, after the perturbation of the coronal plasma by a broadband pulse, the wave front propagates upwards, while the plasma behind it oscillates at the cut-off frequency (see e.g. Lamb 1932; Suematsu et al. 1982; Sych et al. 2012). In a stratified medium, such oscillations at the local cut-off frequency, which are often called trailing oscillatory wakes, could be considered for the interpretation of longer-period compressive waves observed in the corona. Indeed, compressive oscillations with the periods longer than three and five minutes are often observed in the corona, For example, Sych \& Nakariakov (2008) detected $15 \mathrm{~min}$ oscillations above sunspots. The study by Ofman et al. (1997) implies possible fluctuations on longer timescales (20-50 min) high above the limb (1.9-2.45 solar radii). Miyamoto et al. (2014) detected quasi-periodic density disturbances with the period ranging from 100 to $2000 \mathrm{~s}$ at 1.5-20.5 solar radii. The analysis of spectroscopic observations revealed propagating disturbances of about $14.5 \mathrm{~min}$ period in a coronal hole (Gupta et al. 2012). Krishna Prasad et al. (2012) detected 12-25 min periodicities in off-limb fan loop-structures, on-disk plume-like structures, and in the polar plume/interplume regions. Waves of 12, 17, and 22 min periods were found in the loop fans of active regions (Krishna Prasad et al. 2014). Thus, the abundant detection of long-period compressive waves and oscillations in the corona could be attributed to the effect of the slow magnetoacoustic cut-off frequency in field-aligned plasma non-uniformities.

To summarise, we briefly specify the basic results obtained in this study:

- We have constructed a model describing the dynamics of longitudinal waves in vertical, field-aligned coronal plasma structures.

- We have derived the equation describing the propagation of linear long-wavelength slow magnetoacoustic waves in magnetic flux tubes filled in with a stratified plasma of constant temperature.

- The cut-off period for longitudinal waves is found to vary with height, decreasing significantly in the low- $\beta$ plasma and in the plasma with $\beta$ of the order of unity, in contrast with the case of a vertically stratified isothermal atmosphere without magnetic structuring in the horizontal direction.

- The depressions in the cut-off period profiles can prevent the propagation of longitudinal waves along coronal plasma structures towards the higher corona if the periods are longer than these cut-off values that can be as short as fifteen or twenty minutes.

- The long-period (in particular, 15-60 min period) oscillations observed in the solar corona can be the trailing oscillatory wakes induced by broad-band perturbations.

The effect of the significant decrease in the cut-off frequency for slow magnetoacoustic waves in the presence of field-aligned non-uniformities of the stratified plasma demonstrated in this paper requires a detailed follow-up study. 
Acknowledgements. We are grateful to Dr. A.M. Uralov for discussion of the results obtained. The work is supported by the Marie Curie PIRSES-GA-2011295272 RadioSun project, the Russian Foundation of Basic Research under grants 15-32-20504 mol_a_ved and 15-02-01077, and the Federal Agency for Scientific Organisations base project II.16.1.6 No. 01201281652 (ANA), and the STFC consolidated grant ST/L000733/1, and the European Research Council under the SeismoSun Research Project No. 321141 (VMN).

\section{References}

Afanasyev, A. N., \& Nakariakov, V. M. 2015, A\&A, 573, A32

Bakunina, I. A., Abramov-Maximov, V. E., Nakariakov, V. M., et al. 2013, PASJ, 65,13

Berghmans, D., \& Clette, F. 1999, Sol. Phys., 186, 207

Botha, G. J. J., Arber, T. D., Nakariakov, V. M., \& Zhugzhda, Y. D. 2011, ApJ, 728,84

Chorley, N., Hnat, B., Nakariakov, V. M., Inglis, A. R., \& Bakunina, I. A. 2010, A\&A, 513, A27

De Moortel, I. 2009, Space Sci. Rev., 149, 65

De Moortel, I., Ireland, J., \& Walsh, R. W. 2000, A\&A, 355, L23

De Moortel, I., Hood, A. W., Ireland, J., \& Walsh, R. W. 2002, Sol. Phys., 209, 89

De Moortel, I., \& Nakariakov, V. M. 2012, Roy. Soc. London Phil. Trans. Ser. A, 370,3193

DeForest, C. E., \& Gurman, J. B. 1998, ApJ, 501, L217

Deforest, C. E., Hoeksema, J. T., Gurman, J. B., et al. 1997, Sol. Phys., 175, 393

Defouw, R. J. 1976, ApJ, 209, 266

Edwin, P. M., \& Roberts, B. 1983, Sol. Phys., 88, 179

Gupta, G. R., Teriaca, L., Marsch, E., Solanki, S. K., \& Banerjee, D. 2012, A\&A, 546, A93

Hasan, S. S., \& Kalkofen, W. 1999, ApJ, 519, 899

Krishna Prasad, S., Banerjee, D., Van Doorsselaere, T., \& Singh, J. 2012, A\&A, 546, A50

Krishna Prasad, S., Banerjee, D., \& Van Doorsselaere, T. 2014, ApJ, 789, 118
Lamb, H. 1932, Hydrodynamics (New York: Cambridge University Press) Marsh, M. S., De Moortel, I., \& Walsh, R. W. 2011, ApJ, 734, 81

Miyamoto, M., Imamura, T., Tokumaru, M., et al. 2014, ApJ, 797, 51

Musielak, Z. E., \& Ulmschneider, P. 2003, A\&A, 400, 1057

Nakariakov, V. M. 2006, Phil. Trans. R. Soc. A, 364, 473

Nakariakov, V. M., Verwichte, E., Berghmans, D., \& Robbrecht, E. 2000, A\&A 362,1151

Ofman, L., Romoli, M., Poletto, G., Noci, G., \& Kohl, J. L. 1997, ApJ, 491, L111

Ofman, L., Nakariakov, V. M., \& Sehgal, N. 2000, ApJ, 533, 1071

Rae, I. C., \& Roberts, B. 1982, ApJ, 256, 761

Roberts, B. 1985, Phys. Fluids, 28, 3280

Roberts, B. 1991, in Advances in Solar System Magnetohydrodynamics, eds.

E. R. Priest, \& A. W. Hood (Cambridge University Press), 105

Roberts, B. 2006, Phil. Trans. R. Soc. A, 364, 447

Roberts, B., \& Webb, A. R. 1978, Sol. Phys., 56, 5

Roberts, B., \& Webb, A. R. 1979, Sol. Phys., 64, 77

Stepanov, A. V., Zaitsev, V. V., \& Nakariakov, V. M. 2012, Stellar Coronal Seismology as a Diagnostic Tool for Flare Plasma (Weinheim: Wiley VCH Verlag GmbH \& Co. kGaA)

Suematsu, Y., Shibata, K., Neshikawa, T., \& Kitai, R. 1982, Sol. Phys., 75, 99

Sutmann, G., Musielak, Z. E., \& Ulmschneider, P. 1998, A\&A, 340, 556

Sych, R., Zaqarashvili, T. V., Nakariakov, V. M., et al. 2012, A\&A, 539, A23

Sych, R. A., \& Nakariakov, V. M. 2008, Sol. Phys., 248, 395

Thomas, J. H. 1982, ApJ, 262, 760

Van Doorsselaere, T., Brady, C. S., Verwichte, E., \& Nakariakov, V. M. 2008, A\&A, 491, L9

Verwichte, E., Foullon, C., \& Nakariakov, V. M. 2006, A\&A, 449, 769

Vršnak, B., \& Cliver, E. W. 2008, Sol. Phys., 253, 215

Yuan, D., Nakariakov, V. M., Chorley, N., \& Foullon, C. 2011, A\&A, 533, A116

Zajtsev, V. V., \& Stepanov, A. V. 1975, Issledovaniia Geomagnetizmu Aeronomii i Fizike Solntsa, 37, 11

Zhugzhda, Y. D. 1996, Phys. Plasmas, 3, 10

Zhugzhda, Y. D., \& Goossens, M. 2001, A\&A, 377, 330 